\title{
Consecuencias de los desastres naturales en la vida de las personas: síntesis de la literatura y orientaciones para la intervención psicosocial
}

Consequences of natural disasters in people's life: literature review and orientations for psychosocial interventions

ÓsCAR LabR

Ph.D. Profesor Departamento de Desarrollo Humano y Social Universidad de Quebec, dirección electrónica Oscarlabra@uqat.ca Dirección postal: Abitibi-Témiscamingue 445, boulevard de l'Université Rouyn-Noranda (Quebec) Canadá, J9X5E4. Danielle Maltais

Ph.D.Profesora Departamento de Ciencias Humanas Universidad de Quebec, dirección electrónica Damielle.maltai@@uqac.ca Dirección postal Chicoutimi 555 Boulevard de l'Université Chicoutimi, Quebec Canadá, G7H2B1

\section{Resumen}

Este artículo presenta los resultados de un análisis realizado en cerca de 130 publicaciones cientificas, sobre los impactos de los desastres naturales en la salud física y psicológica de personas. El artículo concluye que los efectos negativos de los desastres naturales pueden manifestarse de manera conjunta en varias esferas de la vida, por ejemplo: la salud física, la salud psicológica la vida personal, familiar y social, asi como en lo económico. Las consecuencias de los desastres naturales son distintas tanto a nivel de la salud fisica, la salud psicológica, como sobre el funcionamiento social de los individuos, lo que debe tenerse en cuenta para orientar la labor de los trabajadores sociales que tienen que intervenir en personas siniestradas durante y después de un desastre natural. Los desastres naturales influyen tanto en la forma en la que las personas son afectadas como en el tipo de intervención que debe desplegarse.

Palabras claves. Salud fisica, salud psicológica, consecuencias de desastres, desastre natural, intervención psicosocial.

\section{Abstract}

The article condenses the most significant elements of 130 research articles analyzing the impacts of natural disasters on the physical and psychological health of persons affected by such events The article draws to the conclusion that the negative effects of natural disasters can manifest themselves simultaneously in multiple spheres of victims' lives, including in their physical and psychological health, their personal, family, and social lives, as well as their economic situation social fequen of disasters for physical and psychological health, as well as for thdividuals social functiong, are diverse and must be taken into consid disasters. guiding the interventions of social workers interacting with victims, during and after disasters. Moreover, the distinct charafterics of specific disasters have an incidence on how victims are affected and, consequently

Key words. Physical health, psychological health, consequences of disasters, natural disaster, psychosocial intervention.

\section{Introducción}

Que se trate de una inundación, un huracán, un terremoto, una tormenta de hielo o de una explosión en una fábrica, las consecuencias de este tipo de desastres son distintas para los siniestrados y a comunidad, y varían de una situación a otra en función de varios factores tanto individuales, culturales y sociales. Para Briere y Elliott (2000), así como para Galea y otros (2008), la pérdida de bienes materiales, el miedo a morir o el hecho de sufri 
heridas produce más impacto en las personas que el desastre natural en sí mismo.

Para Charbonneau, Ouellette y Gaudet (2000) una tormenta de hielo no tiene las mismas consecuencias que puede producir un terremoto o una inundación, que pueden durar solo algunos días, pero que producen perturbaciones a través del tiempo en las personas afectadas. La fase de anticipación es cas inexistente en los terremotos y su fase de impacto es bastante corta, contrariamente a una inundación o a una tormenta de hielo. Así, cuando los trabajadores sociales implementan intervenciones de tipo dores socives in caso de desastres naturales, debon naturales, deben tener en cuenta tanto el tipo de desastre (terremoto, inundación, erupción de un volcán, etc.) que constituye en sí un elemento estresante, como las perturbaciones sociales ocasionadas por el siniestro y las consecuencias en la vida de las personas. Esto es sostenido por McFarlane (1995), quien indica que las intervenciones a desarrollar van a variar en función del tipo y gravedad del desastre natural.

La investigación que trata sobre las consecuencias de desastres naturales en la salud biopsicosocia de los individuos es un ámbito muy desarrollado en psicología pero no así en trabajo social (Maltais y Gauthier, 2010; Maltais y otros, 2005; Maltais y otros, 2002: Maltais, Robichaud, Simard, 2001). No obstante, en Quebec (Canadá), debido a aconco obsimiente, en Quebec (Canada), debido a acontecimientos particulares durante estos ultimos años (inundación en la localidad de Saguenay en julio de 1996 y la tormenta de hielo de 1998 en la región metropolitana del gran Montreal, de la Montérégie y en Ontario), varias investigaciones han sido desarrolladas por un equipo de profesores en trabajo social de Quebec (Maltais y Robichaud, 2009; Maltais, 2007; Maltais, Lachance y Brazalete, 2003 2002. Maltais y 2002; Maltais y otros, 2001). Asi mismo, en ambito de la sociologia, distintas han sido las investigaciones que han abordado las consecuencias de los desastres naturales en el funcionamiento socia de organismos comunitarios (Barton, 1969; Miller 2012; Nigg y Tierney, 1993; Rodríguez y Barnshaw, 2006; Stock, 2007; Tierney, 2006).

Los desastres naturales producen consecuencias importantes en la salud biopsicosocial de los individuos (Maltais y Simard, 2008). Por ejemplo, un estudio realizado en Asia por Udomratn (2008) sobre las consecuencias psicosociales de distintos desastres naturales ocurridos en esa región, demuestra que la presencia de estrés postraumático (EPT) en las víctimas varía entre 8,6\% a 57,3\%. Ello dependerí, según este autor, de si se trata de un desastre reciente o no, del tipo de desastre y de su intensidad o fuerza destructora

Existen distintos tipos de catástrofes y los autores tienden a utilizar como sinónimos los términos 作 (ejemplo: un terremoto, una inundación, etc.) o un desastre proveniente de las actividades propias del hombre (accidente tecnológico, guerra, conflictos raciales, etc.) (Maltais, Robichaud et Simard, 2001). En lo concerniente a los desastres naturales, podemos incluir cambios climáticos y meteorológicos bruscos, por ejemplo, las ventiscas, las inundaciones, los huracos o los ciclones, asi cono los ciones, los huracanes o los ciclones, asi como los terremotos (Maltais, 2003). Este tipo de desastres naturales será especialmente objeto de este artículo, dada la frecuencia de ellos en Sudamérica en los últimos quince años.

En un primer momento, se describe la metodología utilizada en los análisis de la literatura que ha sido seleccionada par un segundo momento, se propone una definición del término desastre. Posteriormente, se abordan les elementos siguientes: 1) Consecuencias de los desastres sobre la salud biopsicosocial de los individuos. 2) Factores personales, sociales y culturales de vulnerabilidad en las víctimas de desastres naturales. Finalmente, se presenta una conclusión.

\section{La metodología}

La revisión de la literatura científica se efectuó principalmente a partir de bases de datos informatizadas como: Pubmed, Sociological Abstracts, Medline y Cinahl. Fueron consultados y analizados 130 artículos y/o libros han sido concernientes a las consecuencias psicosociales de los desastres naturales en la salud de los individuos. Los documentos seleccionados para fines de análisis han sido publicados entre 1969 y 2013. Este trabajo estuvo orientado por tres grandes variables que guiaron esta investigación documentaria. Una primera, referida a las consecuencias en la salud física de las personas víctimas de desastres naturales. Una segunda sobre las consecuencias en la salud psicológica de los siniestrados, y finalmente, los factores de vulnerabilidad en las víctimas frente a estos acontecimientos. Los resultados obtenidos a partir de estas tres variables han sido utilizados para fines de reflexión y análisis en intervención psicosocial.

Los criterios de selección de los artículos o los capítulos de libros se acotaron a distinguir textos que se refiriesen específicamente a los impactos psicosociales de los desastres naturales en las personas y que provinieran de estudios tanto cuantitativos como cualitativos realizados en personas expuestas a algún tipo de desastre natural (por ejemplo: terremoto, inundación, temporal de nieve o de hielo, etc.).

Las limitaciones de este trabajo están dadas por la literatura consultada y no es intención de los autores ofrecer un análisis por continente o por tipo de desastre de tales impactos en la salud tanto física como psicológica de las personas. Más bien este trabajo se centró en el objetivo de documentar los impactos psicosociales de los desastres naturales en la salud en general de las personas que han sido víctimas de algún tipo de desastre natural.

\section{Definición del concepto de desastre \\ natural}

Para Bolin (1982), los desastres naturales constituyen una categoría de ac que, de mani intensidad, exponen a los seres humanos a una variedad de tensiones y de rupturas. A este respecto, Barton (1969) en Robichaud y otros (2002: 102) destaca que un desastre natural puede entenderse como una alteración brutal o ruptura en las condiciones de vida de los miembros de un sistema social. Esta ruptura causaría una importante situación de tensión colectiva. En este mismo orden de ideas, Belter y Shannon (1993) mencionan que un desastre puede ser definido por sus características, por su impacto y por la amplitud de los daños causados.

Desde un punto de vista sociológico, un desastre puede entenderse como una situación que forma parte de una categoría más amplia que produce una importante bichaud y otros, 2002). Esta tensión colectiva, sería gatillada cuando varios miembros de un sistema social no reciben el apoyo o condiciones mínimas necesarias de su sistema.

Cuando se trata de estudiar el concepto de desastre, varios autores hacen referencia a la utilización del término acontecimiento traumatizante. Tal es el caso de Solomon y otros, (1987); Norris, Phifer y Kaniasty, (1990). Para la American Psychiatric Asociación (1987), un acontecimiento traumatizante puede comprenderse como un evento «(...) que se sitúa fuera de toda experiencia humana habitual y que puede ser fuertemente estresante para cualquiera (...)» (p. 250)

A principios de los años 80 , un gran número de investigadores dieron una nueva dirección a sus investigaciones, abordando el estudio de los desastres naturales desde una óptica de la vulnerabilidad so- ciopolítica y económica de los individuos. Ello puso en entredicho el paradigma que dominó mucho tiempo en las ciencias sociales. Paradigma que ocultaba las causas profundas de los desastres «dichos naturales» (Gaillard, Liamzon y Maceda, 2007). Asi, los desastres dichos naturales no lo serían nunca totalmente en estado puro, dado que su proliferación es influenciada tanto por el recalentamiento climático como por factores de negligencia humana. De esta forma varios expertos consideran que este fenómeno es también tributario de un conjunto de dificultades estructurales de carácter sociopolílico y económico, donde las consecuencias vividas por las personas tras un desastre dependen de una interacción compleja entre factores individuales sociales. Por lo anterior, es importante abordar el concepto de vulnerabilidad social de los individuos al igual que el concepto de adaptación humana a estrés producido por un desastre natural. A este respecto, parece evidente que factores individuales sociales ejercen una influencia sobre el nivel de vulnerabilidad de distintos grupos sociales expuestos a pérdidas o daños producidos por los desastres naturales (GIEC, 2007; International Strategy for disaster reduction, 2004).

Para el GIEC (2007: 14), «la capacidad de adaptación, intimamente vinculada al desarrollo socioeconómico, se distribuye de forma desigual en la sociedad». En este sentido, el cuarto informe de evaluación del GIEC indicó que, en todas las regiones del mundo, poco importa el nivel de desarrollo algunas subregiones, algunos sectores y algunas colectividades son más vulnerables que otras a los cambios climáticos (Wilbanks y otros, 2007). Este fenómeno es preocupante dado que el número de personas en situación de vulnerabilidad aumenta considerablemente elevando sin cesar los daños materiales y las pérdidas en vidas humanas (International Strategy for disaster reduction, 2004). No obstante, el recalentamiento climático no puede explicarlo todo y no puede ser la única causa del aumento gradual del número de desastres dichos naturales y del número de personas afectadas por estos últimos. Así como lo indican Gaillard, Liamzon y Maceda (2007) en un texto referente a las ciclones ocurridos en Filipinas en 2004, «el discurso contemporáneo en torno al cambio climático (...) contribuye a centrar la atención sobre los riesgos naturales en beneficio de causa más profundas que estarían al origen de los desastres» (p.46).

La frecuencia de los desastres así como la magnitud de las consecuencias humanas, comunitarias, socia- 
les y materiales pueden ser consideradas como: 1) el resultado de la interacción entre distintos factores humanos vinculados a actividades medioambientales inadecuadas (urbanización e industrialización rápida tala de árboles desmesurada construcción en onas de riesgo de inundaciones o desprendimiento de tierras, desregulación industrial, importación de productos tóxicos, etc.); 2) consecuencias de conflictos socioeconómicos (guerras, conflictos políticos, desplazamiento de poblaciones en zonas de riesgo medioambientales); y 3) la distribución desigual de los recursos económicos, sociales culturales entre individuos, comunidades y países (Blaikie, Canon, Davis y Wisner, 1994). Algunos (Blaikie, Cano de estos factores que hacen vulnerables a cierto grupos de individuos, colectividades o regiones son fácilmente identificables. Citemos acá la degradación del medio ambiente o el establecimiento de reagrupaciones humanas en zonas de riesgo. Desgraciadamente, hay otro tipo factores que son menos visibles y que deben ser objeto de estudios sistemáticos, como es el caso de la discriminación racial, la pobreza, la ausencia de derechos cívicos, la distribución desigual de la riqueza, la corrupción o la incapacidad de los gobiernos u organizaciones de proteger y cubrir las necesidades fundamentales de la población (Fédération internationale des Sociétés de la Croix-Rouge et du Croissant-Rouge/ consortium Prevention, 2007)

\section{Consecuencias de los desastres}

naturales en la salud biopsicosocial de las personas

Los estudios tanto cualitativos como cuantitativos referentes a las consecuencias de una inundación que ha a , demuestran que las victimas viven distintas experiencias emocionales (Maltais y Robichaud, 2009). Experiencias que se gatillan en función de las distintas etapas de un desastre, ya sea de pánico (fase de alerta), de la incredulidad, del horror, del terror (fase de impacto), de la desesperanza, de la impotencia (fase de socorro o ayuda), de la pena o del desamparo (fase de restablecimiento) (Maltais y otros, 2005, 2002;

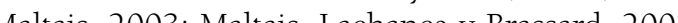
2002; Maltis, Robichaud y Sind, 2001; Lan2002; Maltais, Robichaud y Simard, 2001; Lalande, Maltais y Robichaud, 2000). La presencia de problemas de salud física y psicológica así como de modificaciones en sus hábitos de vida y en sus ac tividades de ocio y de recreación parecen perdurar en el tiempo. En este sentido, citemos la situación lio de 1996 en Saguenay (provincia de
Después de más de dos o tres años de este desastre natural, investigadores han observado diferencias significativas entre siniestrados y no siniestrados, de zonas urbanas y rurales concernientes a problemas de salud psicológica (ejemplo: manifestación de sintomas de ansiedad, de depresión, de EPT de sinionas de ansiedad, de depresión, de EPT y de disfunción social), a la percepción de su estado de salud física, a la frecuencia de sus actividades sociales, así como a su capacidad de responder a las demandes o necesidades de los distintos miembros de su familia (Maltais, Robichaud y Simard, 2001). A este respecto, numerosos son los investigadores que han constatado que la salud biopsicosocial de que hictimas de desastres naturales es fuertemente fuertemente afectada por su exposición a un desastre, tanto por la aparición de fobias, por el sentimiento de muerte inminente, por el miedo de dormirse, como por la presencia de pesadillas recurrentes (Denissen y otros, 2008; Lachance, van Der Kolk y otros, 1996; Santos y Burns, 1994;). Además, el consumo de medicamentos prescritos o no prescritos, de alcohol o drogas parece también ser una de las consecuencias frecuentes de los desastres y esto especialmente en el caso de los hombres (Auger y otros, 2000; Côté, 1996; Green y Lindy, 1994; Maltais y otros, 2002). Por su parte, Maltais, Robichaud y Simard (2001) afirman que, entre todos los problemas de salud psicológica, la presencia de manifestaciones de EPT es el más documentado en los estudios que EPT es el ma presencia de maniestaciones que abordan los efectos de los desastres sobre la salud mental. Los sintomas del EPT comienzan generalmente después de los tres primeros meses seguidos al acontecimiento traumatizante (véase Cuadro $\mathrm{N}^{\circ} 1$ ). Del mismo modo, varios estudios demuestran que una elevada proporción (23-24\%) de los individuos directamente afectados por un terremoto desarrollarán problemas de carácter psicológico, en particular, un EPT (Basoglu, S en particular, un EPT (Basoglu, Salcioglu y Livanou, 2007; Hua, McFarlane y Klimidis, 1988; Wang y otros, 2000). Por su parte Peng y otros (2009), demostraron en su estudio que entre $45 \%$ a $56 \%$ de los individuos de una de las regiones más afectadas por el terremoto de mayo de 2008 en China, habría desarrollado un EPT. En este sentido, Lazaratou y otros (2008) afirman que una persona de dos manifiestan sintomas de EPT durante los seis meses que siguen a un terremoto y que las consecuencias de este último estarían presentes durante más de 50 años después de este tipo de experiencias traumatizantes. Los terremotos pueden entonces producir diversos efectos negativos sobre la salud psicológica de las víctimas. Los estudios demuestran que mientras más se enfrenta a una persona a acontecimientos estresantes producto de un desastre natural, ella tendrá más tendencia a desarrollar un EPT (Chao-Yueh y otros, 2010; Lewin, Carr y Webster, 1998)

A nivel de consecuencias producidas por los desastres naturales en la salud física de las víctimas, los autores concuerdan en que la presencia de desórdenes físicos como el insomnio, las palpitaciones, los temblores, la agitación, la exacerbación de problemas cardiovasculares suelen ser los más recurrentes (Robichaud y otros, 2002, Friedman et Shnurr, 1995; Aptekar, 1994). Durante los días o las semanas siguientes al periodo post-impacto, los síntomas físicos pueden manifestarse bajo diferentes formas, como vómitos, diarreas, dolores de cabeza o dificultades respiratorias (Raphael, 1986)

Los desastres naturales pueden también generar distintas consecuencias en los individuos y en las colectividades, consecuencias que no son solamente de carácter físico y psicológico. Así, algunos estudios han demostrado que las prácticas de vida, las actividades de ocio, la vida marital, familiar y social de la víctimas, el rendimiento laboral, las capacidades de satisfacer económicamente a la familia y la autopercepción pueden también ser profundamente alteradas después de una exposición a un desastre natural (Bolin, 1982; Crabbs y Black, 1984; MacDonnell y otros, 1995; Maltais y otros, 2002: Maltais, Robichaud et Simard, 2001; Ticehurst et otros, 1996). Por otro lado, no son solamente los acontecimientos desencadenados durante y después de un desastre natural los que aumentan la vulnerabilidad de los individuos

CUADRO N 1

CRITERIOS DIAGNÓSTICOS DE TRASTORNOS POR ESTRÉS POSTRAUMÁTICO (TEPT)

\begin{tabular}{|c|c|}
\hline Criterios diagnósticos & Mínimos requeridos \\
\hline \multirow{2}{*}{ A. Haber vivido un evento traumático } & Al. Amenaza a la vida o a la integridad física. \\
\hline & A2. Miedo, impotencia, horror. \\
\hline \multirow[b]{2}{*}{ B. Re-vivencias del evento } & 1 sintoma sobre 5 posibilidades: \\
\hline & $\begin{array}{l}\text { Recuerdos, sueños, flashbacks, angustia psicológica y fisiológica } \\
\text { en presencia de elementos asociados a un trauma. }\end{array}$ \\
\hline & 3 sintomas sobre 7 posibilidades: \\
\hline C. Evitación persistente y reacciones menos agudas & $\begin{array}{l}\text { Evitación de pensamientos, sentimientos, actividades, lugares } \\
\text { asociados al trauma, amnesia parcial del evento, disminución } \\
\text { del interés, sentimiento de desapego, restricción de afectos, } \\
\text { sentimiento de un futuro oscuro. }\end{array}$ \\
\hline \multirow[b]{2}{*}{ D. Hiperactivación } & 2 sintomas sobre 5 posibilidades: \\
\hline & $\begin{array}{l}\text { Dificultades para dormir, irritabilidad, dificultades de } \\
\text { concentración, ansiedad creciente, sobresaltos. }\end{array}$ \\
\hline E. Duración de un mes & Los sintomas duran a lo menos un mes. \\
\hline $\begin{array}{l}\text { F. Sufrimiento significativo y alteración del } \\
\text { funcionamiento }\end{array}$ & $\begin{array}{l}\text { Angustia y secuelas en las esferas significativas de la vida del } \\
\text { individuo. }\end{array}$ \\
\hline
\end{tabular}

APA (1994) frente a posibles problemas de salud biopsicosociales. En efecto, los acontecimientos principales, llamados factores pre-dispositivos (prétraumáticos) Brillon, 2002) que ocurren seis meses antes de un desastre, desempeñarían un papel importante en el desarrollo de problemas de salud post-desastre, en particular, por lo que se refiere a la probabilidad de desarrollar un EPT (Lewin, Carr y Webster, 1998). A este respecto, los factores que hacen más vulnerables a las personas frente a un desastre natura pueden agruparse en distintas categorías: la demografía social (por ejemplo la edad, clase social, pertenencia étnica y educación de la población), el acceso a informaciones exactas sobre un desastre y las capacidades de resiliencia de la comunidad. Es pertinente, o incluso esencial, situar en el tiempo y el espacio, algunos factores de riesgo vinculados a las consecuencias psicosociales de un desastre natural. Estos factores de vulnerabilidad se presentan

\section{os factores de vulnerabilidad}

Los factores de vulnerabilidad son agrupados en cuatro secciones: 1) La edad de las víctimas, 2) las desigualdades sociales, 3) la influencia de la cultura, y finalmente 4) las actividades socializantes.

\section{Co edad de las victimas}

Concerniente a la edad de las víctimas de desastres naturales, las investigaciones demuestran que os niños y los adolescentes serían una población Hovington, Maltais y Lalande, 2002; Olson, 1993 especialmente vulnerable (Crabbs y Heffron, 1981; 
al igual que las personas de 65 años o más (Gignac, Cott y Badley, 2004; Norris y otros 2002; Wang 2007). Los desastres naturales pueden afectar así a conjunto de los integrantes del grupo familiar y la presencia de problemas de salud de tipo psicológipresencia de probens de salud de tipo psicologicillar ciertos raumas de tipo secundario en sus cercanos, especialmente en los niños (Pynoos y Nader, 1993) Para Chao-Yueh y otros (2010), los niños que son expuestos a desastres naturales tienen un mayor riesgo de desarrollar un EPT. Newman (1976), uno de los pioneros en interesarse en la salud psicológica de los niños que han vivido aloún tipo de desastre naturat, considera que las consecuencias de estos eventos en la salud de los infantes depende de tres factores: a) el nivel de desarrollo del niño, b) su percepción sobre la respuesta que la familia otorga al desastre, y c) el nivel de exposición del niño al desastre. Por su parte, Lystad (1984) indica que los niños no reaccionan a una experiencia traumatizante o a un desastre como individuos aislados, el contexto medioambiental compuesto por los patre c la red f miliar adquiere un sentido importeres y la red familiar adquere un sentido importante. El hecho de constatar la vulnerabilidad de sus padres y la ausencia de control ante los desastres pued constituir en los infantes una fuente de ansiedad as como un sentimiento de inseguridad (Shelby y Tredinnick, 1995)

Análisis de regresión indican que la exposición prolongada a un desastre natural, el apoyo parental, los conflictos familiares y la protección excesiva predicen los síntomas del EPT en los niños. Además, una exposición prolongada a un desastre, un nivel de escolaridad y un estatus socioeconómico medio de los padres son predictores de síntomas de EPT (Kar y otros 2007). La edad cognoscitiva de los niños predice también los síntomas del EPT (Jolian y otros (2008) argumentan que la exposición prolongada al desastre está asociada a bajos niveles de satisfacción de vida. Los niños con un EPT son más vulnerables que el resto a presentar problemas de retraso en su desarrollo (Delamater y Applegate, 1999). En este sentido, Russoniello y otros (2002), muestran en su estudio realizado en víctimas del huracán Floyd, que $71 \%$ do los vinfates presenta sintomas de EPT moderados. son dos veces más propensas a informar de síntomas del EPT que los niños. Además, Jones y otros (2001) constataron en un estudio realizado en infantes que vivieron el huracán Andrew, que el nivel de estrés de las niñas de enseñanza básica es más elevado que en los niños realizado en niños que vivieron el huracán Katrina, demuestran que el $88,6 \%$ presenta al menos un desorden co-mórbido (por ejemplo: problemas de ansiedad o de desconfianza extrema).

Otros estudios indican que de 6 a 12 meses posteriores a un desastre, los niños no muestran disminución significativa de síntomas de EPT (Lack, 2006). Según Pullins y otros (2005), la severidad de la exposición y el estrés psicológico se asocian significativamente a los síntomas del EPT informados por los niños. Un resumen de investigación que trata especificamente del terremoto de Chile de 2010 menciona que un $90 \%$ de los jóvenes entrevistados entrevistados mostraban señales de traumatismo un mes después del desastre (Martín y otros, 2010). El hecho de que el terremoto se haya producido durante la noche influyó probablemente en la frecuencia y la intensidad de algunos síntomas. En efecto, más de la mitad de estos jóvenes de entre 12 a 15 años afirmaron que se despiertan en la noche o que presentan pesadillas (Martín y otros, 2010)

Por otra parte, Kozlovskaia y otros (1991) observaron en un estudio sobre los impactos de un terremoto en Armenia realizado en 1.900 niños menores de diez años y 100 miembros de sus familias, que el principal factor patógeno que produjo desórdenes neuropsiquiátricos en los infantes fue el efecto sismico del terremoto y el t mental asociado a los momentos de ocurrido del desastre natural. Además, los investigadores observaron fuertes perturbaciones emocionales durante las semanas que siguieron al terremoto, en la mayoría de los niños que vivían cerca del epicentro sísmico. Un cuarto de los adolescentes sufrió de fobia (por ejemplo: miedo a que ocurra un nuevo terremoto)

En las personas de 65 años o más, los estudios sostienen que este grupo presenta un elevado riesgo de morir o sufrir heridas tras su exposición a un desastre natural. Por lo tanto, los adultos mayores se reconocen como muy vulnerables durante y después de un desastre natural (Fernández y otros, 2002; Knowles y Garrison 2006; Maltais y Gauthier, 2007; Rosenkoetter y otros 2007; Stephens y otros, 2007; Spiegel, 2006). Los adultos mayores, en especial los que presentan incapacidades físicas o cognitivas que conlleven una pérdida de autonomía (previa al desastre), forman así parte de los grupos de riesgo de sufrir más cantidad de heridas, de morir o de presentar un alto nivel de estrés postdesastre (Gignac, Cott y Badley 2004. Maltais, Côté y Gauthier, 2007). Thompson y otros (1993), argument el aumento de la edad está ligado a un aumento en la vulnerabilidad psicológica luego de una exposición a un desastre natural.

En otro estudio, Chan y otros (2003) así como Osaki y Minowa (2000) demostraron que en los terremotos las mujeres y las personas mayores presentarían un importante riesgo de morir al año siguiente del un importante riesgo de morir al año siguiente del
desastre, al igual que las personas con algún tipo de invalidez física. Kovats y Ebi (2006), destacan que las personas de 65 años o más presentan aún mayor riesgo de morir tras una ola de calor extrema, debido a varios factores sociales como el hecho de vivir solas, de ser aisladas socialmente, de poseer un sistema de climtización defecturo, asi como vivir en un departamento situado en el piso más alto de un edificio.

Ticehurst y otros (1996), en un estudio en el que comparan las consecuencias psicosociales de un terremoto en Australia entre personas menores y mayores de 65 años, demuestran que estas últimas declaran presentar más síntomas asociados al EPT. Por su parte, los resultados del estudio de Tyler y Por su parte, los resultados del estudio de Tyler y
Hyott (2000) realizado en 651 personas de 65 años o más, siniestrados en las inundaciones de lowa en los Estados Unidos, remarcan una asociación positiva entre la presencia de manifestaciones depresivas antes de una exposición a un desastre natural y la presencia de estas manifestaciones después de este tipo de desastre. El sentimiento de según el gencro, la pententilidad de informaciones de la parte de la comunidad y la capacidad de resiliencia de esta última (Hutton, 2004). Según un estudio de Ticehurst y otros (1996), a pesar que las personas de 65 años o más declaran presentar más síntomas correspondientes a un EPT que los jóvenes, los adultos mayores declaran vivir en menor cantidad de situaciones amenazantes o de perturbaciones.

A pesar de que son numerosos los estudios que demuestran que las personas de 65 años o más resultan más afectadas que los jóvenes frente a un desastre natural (Carr, y otros, 1997; Krause, 1987; Lewin, Carr y Webster, 1998; Logue, Ollendick y Hoffman, 1982. Melick y Stuening 1981. Miller Turner Kimbal, 1981; Phifer y ;icehurst y otros, 1996;), otros estudios sostienen que los adultos mayores serían menos afectados que otros grupos etarios (Bolin y Klenow, 1982-1983; Finnsdottir y Elklit, 2002; Green y otros, 1996; Hutchins y Norris, 1989; Kato y otros, 1996; Knight, Gatz, Heller y Bengtson, 2000: Norris y Murrel, 1988; Thompson, Norris y Hanacek, 1993; Tyler y Hoyt, 2000; Weintraub y Ruskin, 1999). En este sentido, resultados simila- res fueron encontrados por Acenio y otros, (2006) en una investigación realizada en 1.130 personas mayores de 60 años y en 413 jóvenes adultos, residentes en 33 condados de Florida. En este estudio as personas mayores de 60 años informan de pocos síntomas de EPT, de desórdenes depresivos o de desórdenes de ansiedad generalizada. Para Kohn y otros (2005), no habría diferencia significativa en la prevalencia del EPT en las personas mayores de 65 años y en los jóvenes adultos. Otros investigadores mencionan también que los adultos mayores no se perciben como estando más afectados a largo plazo en cuanto a la salud física, comparativamente a los dultos más jóvenes (Maltais, Robichaud y Simard, 2001)

Para algunos investigadores, el riesgo de desarrollar secuelas después de una exposición a un desastre natural se asociaría más bien al tipo de personalidad de la persona siniestrada que a su edad propiamente tal. De esta forma, algunas características de personalidad aumentarían el riesgo de desarrollar un EPT después de un desastre natural. A este respecto, Chao-Yueh y otros (2010); Lewin, Carr y Webster (1998) remarcan la vulnerabilidad de la persona de tipo neurótica, introvertida y aquella con una baja autoestima. Contrariamente, los riesgos serían menores para las personas extrovertidas. Eso quizás debido al hecho de que estos últimos tienden más a exteriorizar lo que experimentan o sienten y a buscar ayuda cuando ello es necesario. Además, algunos mecanismos de protección como el optimismo disminuirían el impacto de las consecuencias negativas en las víctimas (Ahmad, 2010).

\section{Las desigualdades sociales}

Las consecuencias psicosociales de los terremotos varían también según las características individuales y sociales de las personas afectadas como: el ingreso familiar y el nivel de educación (Finnsdottir y Elklit, 2002; Hutton, 2004; Smith, 1996), un apoyo social precario (Lee y otros, 2004) así como la presencia de problemas de salud mental (Caldera y otros, 2007). Así, tenemos que no solamente variables de tipo individual influyen en la forma en que las personas se enfrentan a un desastre natural. Factores de orden macrosocial deben también tenerse en cuenta cuando se trata de estudiar las consecuencias de los desastres naturales en la salud física y psicológica de las víctimas, y las desigualdades sociales son un claro ejemplo. A este efecto Gupta y Agrawal (2010), mencionan que las personas más vulnerables (antes de ocurrido un terremoto) - que se trate de las mujeres, los niños o las personas viviendo 
con VIH, etc. - deben hacer frente situaciones aún más deplorables después de un desastre. Ya que presentan condiciones de vida más precarias que la media, estas personas son más propensas a desarrollar distintos problemas de salud después de un desastre natural. Además, la situación a menudo caótica generada por un desastre natural hace que estas personas sean muy vulnerables a la violencia, la explotación, a las agresiones sexuales, etc. A este respecto, Zakour y Harrel (2003) consideran que las personas en condiciones de pobreza tienen poco acceso a servicios de apoyo debido a la injusticia social de la cual son víctimas, en particular a una distribución desigual de los recursos a las comunidades. Existe también una presencia de factores de riesgo más importantes, en particular, el mal estado de infraestructuras colectivas (carreteras, puentes servicios de acueducto), así como la presencia de barreras geográficas que retrasan o hacen más lenta la distribución de la ayuda en los barrios o sectores desfavorecidos (Zakour et Harrel, 2003).

Así, las consecuencias individuales son variables según el estatus socioeconómico de la población. Para Oliver-Smith y (1990); Logue, Melick y Struening (1981), el restablecimiento de los siniestrados es la mayor de la veces directamente proporcional a su estatus social. Algunos autores mencionan también una intensificación de las desigualdades sociales después de un terremoto (Ng y otros, 2009). En este sentido Peng y otros (2009), señalan que el riesgo de desarrollar un EPT es más elevado en las minorías étnicas. Este fenómeno está directamente vinculado a las desigualdades sociales puesto que las minorías son generalmente más propensas a vivir problemas a nivel social y económico en comparación al grupo mayoritario.

\section{La influencia de la cultura}

La cultura influye de manera particular en la manera de vivir o de enfrenar la pérdida de un familiar víctima de un desastre natural. Por ejemplo, para los haitianos practicantes del vudú, no significa solamente haber perdido a uno o más de los suyos, es también el hecho de no poder proporcionarles una ceremonia funeraria conveniente (Schininà y otros, 2010), lo que no sería distinto para la población chilena, de fuertes raíces católicas. La situación caotica generada por el Sismo del 12 de enero de 2010 en Haití, no permitió cumplir con esta tradición, lo que aumentó el nivel de estrés y angustia de algunas personas ya vulnerables (Schininà y otros, 2010) Lo mismo en el caso de las víctimas del tsunami de 2010 en la ciudad de Constitución, Chile.
Por otra parte, ciertos elementos de la cultura pueden favorecer los intercambios, la expresión de emociones negativas y hacer disminuir el EPT causado por un desastre natural (Schininà y otros, 2010). Por ejemplo, la cultura vudú concede una gran importania a la danza y a las pecusiones. dos pueden exteriorizar sus emociones utilizando estas son de expresión que forma parte integrante de su cultura. No obstante lo anterior, para Smith (1996) la práctica religiosa y el vivo ardor que la caracteriza estaría asociado a un mayor abandono psicológico de la persona.

Mencionemos que en ciertas culturas, la religión desempeña un papel fundamental en la comprensión y en la interpretación de los desastres naturales. El desastre puede verse como procedente de una voluntad divina. Ello fue observado en el estudio de Labra, (2013) en adultos de la ciudad de Talca que vivieron el terremoto del 27 de febrero de 2010. Según Schininà y otros (2010), en la India la muerte es asociada a un estado de normalidad del ser humano, ya que la muerte toma un «sentido o una razón de ser». Esta manera de percibir la muerte consecuencia de un desastre natural no es distante del dolor que ello implica en sus vidas. Ahora bien, todas las culturas han desarrollado mecanismos de defensa contra la muerte y contra la angustia de la muerte, y han intentado superar o vencer este miedo que ella produce en el ser humano. Según Gendron y Cantero (1997), la preocupación excesiva sobre la vida y la muerte biológica ha llegado a borrar la dimensión espiritual de la vida y la muerte como experiencia psíquica. Lo anterior es lo que el «movimiento de redescubrimiento de la muerte» pretende rehabilitar. En este sentido, para Turner (1979) en Gaudreau (2002), «el enfoque del mundo es típicamente intervencionista, no fatalista $y$ orientado hacia la acción, de modo que la respuesta cultural a los desastres naturales va intentar actuar sobre ella y así organizarse para intentar contradecir sus efectos» (p.32), lo que para Gaudreau vendría a mostrar el carácter de normalidad otorgado al desastre natural.

\section{Las actividades socializantes}

Según un estudio de Wang y otros (2000), el hecho de ser afectado o no por un desastre natural no influye mucho en las relaciones sociales de los individuos. Una persona que tiene una vida social activa antes de un desastre, tendrá tantas relaciones interpersonales antes como después de tal acontecimiento. Por otro lado Maltais y Côté, (2007); Maltais (2003), indican que algunas personas pueden vivir el aislamiento a raíz de un desastre natural. Por ejemplo, la pérdida de uno prójimo puede afectar de forma considerable la red social de un individuo y especialmente aquella de los adultos mayores (Maltais y Côté, 2007).

La reconstrucción de un barrio, de una región afectada, así como la situación personal de las víctimas de un desastre natural necesita de la movilización de un número considerable de individuos para que vuelva a su total normalidad. Algunas personas deben dejar de lado o conceder menos importancia a sus relaciones interpersonales cotidianas. Así por ejemplo, un adulto mayor podrí recibir menos viejemplo, un a sitas de los miembros de su familia después de un desastre debido a las obligaciones que ello genera en sus hijos o en los mismos siniestrados. En este sentido Chao-Yueh y otros (2010), indican que la situación de reducción de las actividades sociales después de un desastre natural es un factor que aumentaría el riesgo de EPT a corto y a largo plazo. 位 de él) después de un desastre natural aumentaría el riesgo de desarrollar un EPT u otro problema psicológico (Chao-Yueh y otros, 2010; Ng y otros, 2009). Al contrario, la presencia de una red social en torno a las víctimas les ayuda a enfrentar mejor las consecuencias de un evento traumatizante. En este sentido, Schininà y otros, (2010) mencionan que después del terremoto de 2010 en Haití, ha habido una revitalización del «lakou». Este término involucra a la familia inmediata y a la familia exteninvolucra a la familia inmediata y a la familia exten-
dida, donde todos viven bajo el mismo techo. Este «lakous» habría sido un factor determinante de resiliencia para la población haitiana. A este respecto, la Organización Internacional para las Migraciones habría mencionado su interés en utilizar, después del terremoto los slahousw durante la relocalización de la población, con el fin de garantizar una mayor protección y apoyo a las personas más vulnerables. El carácter cultural específico de este fenómeno dificulta quizás la transmisión de esta forma «de hacer» en otras culturas distintas a la haitiana, como alternativa para disminuir las consecuencias psicosociales negativas en las víctimas de desastres naturales

Entre los estudios consultados sobre las consecuencias de los desastres naturales, no todas las investigaciones concluyen necesariamente que hay resultados negativos en la vida de las personas. En este sentido, Vázquez y otros (2001) observan una presencia de emociones positivas posterremoto. Por ejemplo, a pesar de los impactos de este tipo de acontecimientos, la mayoría de las persona capaces de vivir momentos de alegría durante los tres meses seguidos a un desastre natural. La gente representa estos momentos como la alegría de estar aún con vida y el sentimiento de estar rodeado por los suyos (Vásquez y otros, 2001). Las víctimas de desastres pueden también hacer aprendizajes posiivos relativos, por ejemplo, a sus capacidades de reacción frente al desastre o a sus relaciones interpersonales (Labra, 2013; Maltais y Gauthier, 2010).

\section{La intervención psicosocial en caso}

\section{de desastre}

Les desastres movilizan generalmente una diversidad de profesionales, durante un período de tiempo variable, que puede ir de algunos días a varios meses. Estos actores deben ayudar a las víctimas y a los miembros de sus familias en distintos lugares públicos y privados, diagnosticando al mismo tiempo a las personas en riesgo de presentar problemas de salud biopsicosocial como el EPT u otros desordenes psicológicos. En este sentido, la intervención de crisis en caso de desastre exige conocimientos específicos que los profesionales no adquieren necesariamente en el marco de su formación académica (Maltais, 2008, 2006; Maltais y Rheault, 2005). Destinados a intervenir a la vez sobre varios frentes, los profesionales efectúan generalmente distintas tareas de apoyo moral y técnico que permiten a las víctimas manifestar su dolor, sus angustias y desarrollar estrategias adecuadas de adaptación para la disminución de los problemas de salud físicos o psicológicos postdesastre. Algunos profesionales tendrán que acompañar a los sobrevivientes en la identificación de cadáveres, otros tomarán a cargo los centros de atención y alberges. Todos tendrán que poner en práctica sus conocimientos y sus habilidades en «intervención de urgencia» para trabajar con personas emocionalmente muy afectadas (Maltais y Rheault, 2005)

Mencionemos que la asistencia social se relaciona históricamente con la atención de víctimas en caso de desastres. Por su parte, la investigación y la intervención en los ámbitos micro y macro social concerniente a las consecuencias y los impactos de los desastres se ajustan a la misión del trabajo social (Zakour, 1996). Streeter y Murty (1996) consideran que históricamente se ha implicado a los trabajadores sociales en la ayuda a las víctimas cuando se produce un desastre. No obstante la contribución de los trabajadores sociales en este ámbito, su aporte es generalmente muy poco reconocido (Zakour, 1996). En caso de desastre, los trabajadores sociales, por ejemplo, tienen que evaluar el estado de salud psi- 
cosocial de las personas evacuadas, coordinar las actividades de apoyo a las personas que presentan dificultades de adaptación, visitar y reconfortar a las víctimas y sus prójimos en distintos lugares, asistir a los siniestrados en sus trámites ante las autoridades públicas o facilitar la creación de comités de apoyo a las víctimas y a los miembros de su familia. De manera preventiva y con el fin de proteger la salud de la población, los trabajadores sociales pueden además acompañar a los individuos en su lucha contra la contaminación medioambiental o contra cualquier otro fenómeno que aumente los riesgos de catástrofes tecnologicas (Maltais, 2008,2006).

\section{Conclusión}

Esta síntesis referente a los impactos de los desastres naturales en la salud física y psicológica de las personas expuestas a un desastre natural, permite constatar que las consecuencias de tales eventos la esencia misma de la exisencia humana de los individuos y en particular de aquellos que se encuentran en condiciones de vid más vulnerables antes de su exposición a los desastres naturales. Aunque los desastres naturales son fuentes de tensión que perturban las responsabilidades sociales, familiares e individuales, constituyen al mismo tiempo un potencial para reconsiderar vida y motivar a los individuos a prestar más tiempo $\mathrm{y}$ atención a sus seres queridos.

Los resultados de las investigaciones consultadas demuestran que los efectos negativos de lo desastres naturales pueden manifestarse simultáneamente en varias esferas de la vida de la persona, o sea: la salud psicológica, la salud física, la vida personal, fam diciones económicas. A este respecto, se podría decir que estos acontecimientos negativos vividos podrían tener una incidencia importante en la forma en que la gente ve o percibe su futuro. Las consecuencias sobre la salud física y psicológica son reales y deben tenerse en cuenta para guiar la labor de los trabajadores sociales que tienen que inervenir ante los siniestrados un desarte ntural. Estos impactos yo son uniformes en la población, de ahí la importancia de tener en cuenta las condiciones de vida de las víctima y sus capacidades tanto físicas, psicológicas, sociales o financieras para sobreponerse y hacer frente a los distintos estreses asociados a la pérdida de sus bienes o de seres queridos ocurrido durante un desastre. Además, la naturaleza del desastre influye en la forma en que las personas son afectadas, as como en la forma en que la intervención debe des- plegarse con el fin de reducir los efectos negativos. Por otro lado, la pérdida de sus bienes, la pérdida de un próiimo, la deslocalización no serían más que algunos ejemplos de incidencias concretas de los desastres naturales en la vida de las victimas. Dado que algunos tipos de desastres naturales no pueden evitarse, es primordial comprender la dinámica que rodea las consecuencias psicosociales de estos acontecimientos en los individuos, y en las colectividades y desarrollar distintos tipos de intervenciones sociales tanto predesastre como postdesastre en función del nivel de vulnerabilidad de los individuos, de las pérlide vufieras aś como de las perurbaciones sociales causadas por el siniestro. Para Sundet y Mermelstein (1996) la intervención psicosocial debería priorizar a lo menos cinco grandes tipos de acciones: 1) la puesta en marcha de coaliciones entre los distintos organismos comunitarios 2) favorecer un apoyo natural y mutuo entre los individuos de la comunidad 3) la instauración de estrategias de comunicaciones y contactos entre los líderes locales para que estos comprendan y asimilen claramente sus tareas y sus competencias 4) la formación de los distintos funcionarios de instituciones gubernamentales o de voluntarios de la comunidad para que estos se familiaricen con la intervención en situación de desastre y 5) el establecimiento de planes urgencia en cada una de las comunidades, con el fin de anticipar los acontecimientos traumáticos y evitar las crisis masivas.

Los trabajadores sociales tienen que intervenir ante varios actores sociales, tanto antes, como durante y después de un desastre natural, con el fin de garantizar que las necesidades de todos los individuos, y especialmente que las necesidades de los más vulnerables sean atendidas.

Las formas y las modalidades de sus intervenciones deben adaptarse a las circunstancias, a las distintas dificultades vividas por las víctimas y a las consecuencias que tienen los desastres sobre las personas afectadas: pérdida de un familiar o un cercano, de bienes esenciales para su seguridad, etc. En caso de desastre, los trabajadores sociales deben entre otras cosas visitar los siniestrados, escucharlos y prestar una atención especial a las personas que presentan emociones negativas (sentimiento de abandono, de desesperanza, angustia, ansiedad, etc.). Deben también favorecer la ayuda mutua entre pares y poner en contacto a las víctimas con los recursos necesarios disponibles en la red social

\section{Bibliografía}

ACERNIO, R. et al. (2006). Risk and Protective Factors for Psychopathology Among Older versus Younger Adults After the 2004 Florida Hurricanes, American Journal of Geriatric Psychiatry, 14(12), 1051-1059.

AHMAD, FEDER A., LEE, E., WANG, Y. et al. (2010). Earthquake Impact in a Remote South Asian Population. Psychosocial Factors and Posttraumatic Symptoms. Journal of Traumatic Stress, 23(3), 408-412.

AMERICAN PSYCHIATRIC ASSOCIATION (1994), Diagnostic and Statistical Manual of Mental Disorders. Washington, DC: American Psychiatric Association.

AMERICAN PSYCHIATRIC ASSOCIATION, (1987). Diagnostic and Statistical Manual of Mental Disorders (DSM). Washington, DC: American Psychiatric Association

APTEKAR, L. (1994). The Psychology of Disaster Victims, en L. Aptekar (ed.), Environmental Disaster in Global Perspectives, New York, G.K., Hall et Co., 79-126.

AUGER. PL., VERGER, P., DABM W., GUERRIER, P., et al. (2000). Sinistres naturels et accidents technologiques. En: Environnement et santé publique - Fondements et pratiques, pp. 517-535. Gérin M, Gosselin P, Cordier S, Viau C, Quénel P, Dewailly É, rédacteur. Edisem/ Tec $\&$ Doc, Acton Vale /Paris.

BARTON, AH. (1969). Communities en Disaster: a Sociological Analysis of Collective Stress Situations, Doubleday \& Cie, New York, Anchor Books.

BASOGLU, M., SALCIOGLU, E., M. LIVANOU (2007). A randomized controlled study of single-session behavioral treatment of earthquake-related post-traumatic stress disorder using an earthquake simulator. Psychological Medicine, 37, 203-213.

BELTER, RW., \& MP. SHANNON (1993). Impact of Natural Disaster on Children and Families, en F.S. Conway (ed.) Children and Disasters, South California, Charleston, 85-103.

BLAIKIE P., CANNON T., DAVIS I., \& WISNER B. (1994). At Risk: Natural Hazards, People’s Vulnerability, and Disasters, Routledge, New York.

BOLIN, R. (1982). Long-term Family Recovery Disaster, Colorado, Library of Congress.

BOLIN, R., D. J. KLENOW (1982-1983). Response of the Journal of Aging Human Development, 16, 283-296.

BRIERE,J. \& D. ELLIOT (2000). Prevalence, Characteristics, and Long-Term Sequelae of Natural Disaster Exposure in the General Population. Journal of Traumatic Stress. 13(4), 661-679.

BRILLON, P. (2002). Diagnostic et traitement cognitivocomportemental du trouble de stress post-traumatique, en D. Maltais (dir.) Catastrophes et état de santé des individus, des intervenants et des communautés. Saguenay, GRIR, UQAC, 69-97
CALDERA, T., PALMA, L., PENAYO, U., \& G. KULLGREN (2001). Psychological impact of the hurricane Mitch in Nicaragua in a one-year perspective, Social Psychiatry and Psychiatric Epidemiology, 36(3), 108-114

CARR, VJ., TJ. LEWIN, RA. WEBSTER \& J. KENARDY (1997). A Synthesis of the Findings from the Quake Impact Study: A Two-year Investigation of the Psychosocial Sequel of the 1989 Newcastle Earthquake, Internationa Journal of Social Psychiatry and Psychiatric Epidemiology 32, 123-136.

CHAN, CC., YP. LIN, HH. CHEN, TY. CHANG, TJ. CHENG $\&$ LS. CHEN (2003). A population-based study on the inmediate and prolonged effects of the 1999 Taiwa earthquake on mortality, Annals of Epidemiology, 13 ,
502-508.

CHAO-YUEH, S., T. KUAN-YI, et al. (2010). A threeyear follow-up study of the psychosocial predictors of delayed and unresolved post-traumatic stress disorder in Taiwan Chi-Chi earthquake survivors. Psychiatry \& Clinical Neurosciences, 64(3), 239-248.

CHARBONNEAU, J., F-R. OUELLETTE ET S. GAUDET (2000). Les impacts psychosociaux de la tempête de verglas au Québec. Santé Mentale au Québec, 25(1), 138-162.

CRABBS, M.A.ET K.U. BLACK (1984). Job Change following a Natural Disaster. The Vocational Guidance Quarterly, 32(4), 232-239.

CRABBS, MA., ET E. HEFFRON (1981). Loss Associated with a Natural Disaster, Personnel and Guidance Journal. $59,378-382$

CÔTÉ L. (1996). Les facteurs de vulnérabilité et les enjeux psychodynamiques dans les reactions post-traumatiques. Santé Mentale au Québec. 21(1), 209-228.

DAVID, D., et al. (1996). Psychiatric morbidity following Hurricane Andrew. Journal of Traumatic Stress, 9(3),

DELAMATER, AM. \& EB. Applegate (1999). Child Development and Post-traumatic Stress Disorder after Hurrican Exposure. Traumatology, 5(3), 20-27.

DENISSEN, J.A., L. BUTALID, L. PENKE \& VAN AKEN MAG. (2008). The effects of weather on daily mood: a multilevel approach. Emotion, 8(5), 662-667.

FÉDÉRATION INTERNATIONALE DES SOCIÉTÉS DE LA CROIX-ROUGE ET DU CROISSANT-ROUGE/ CONSORTIUM PROVENTION (2007). Analyse de vulnérabilité et de capacités, Outils d'intégration de la réduction des risques de catastrophes, Note d'orientation 9 . www.proventionconsortium.org/themes/default/pdf/tools

FERNÁNDEZ. LS., D. BYARD, C.C. LIN, S. BENSON \& JA. BABERA (2002). Frail Elderly as Disaster Victims Emergency Management Strategies, Prehospital and Disaster Medicine, 17 (2), 67-74.

FINNSDOTTIR, T. \& A. ELKLIT (2002). Posttraumatic sequel in a community hit by an avalanche. Journal of
Traumatic Stress, 15(6), 479-485. 
FRIEDMAN, J.J. \& P.P. SCHNURR (1995). The relationship between Trauma, Post-traumatic Stress Disorder, an Physical Health, en M.J. Friedman, D.S. Cherney et A.Y. Deutch (eds), Neurobiological and Clinical Consequences of Stress: from Normal Adaptation to Post-traumatic Stiess

Disorder, Philadelphia, Lippincott-Raven, 507-524.

GAILLARD, J-C., LIAMZON, C.C., MACEDA, E.A. (2007) Retour sur les causes d'une catastrophe. pourquoi plus Développement, 137(1), 35-50

GALEA, S. et al. (2008). Financial and social circumstances and the incidence and course of PTSD in Mississipp The years after Hurricane Katrina. Journel of Traumatic Stress, 21(4), 357-68.

GIEC (2007). Bilan 2007 des changements climatiques. Contribution des Groupes de travall l, II et III au quatriem Rapport devaluation an Groupe dexpertsintergouvernement sur l'évolution du climat [Equipe de redaction principale, Pachauri, R.K. et Reisinger, A. (publié sous la direction de )]. GIEC, Genève, Suisse, 103 pages.

GIGNAC, M.A., C. COTT, C.A. \& E.M. BADLEY (2004). Living with a Chronic Disabling Illness and Then Some: Data from the 1998 ce Storm. Canodisn Journt on Aging 8 Ice Storm. Canadian Journal on Agin

GREEN, B.L., J.C. GLESER, J.D. LINDY, M.C. GRACE \& A.C. LEONARD (1996). Age Related Reactions to the Buffalo Creek Dam Collapse, en P.E. Ruskin et J.A. Talbo (Eds), Aging and Posttraumatic Stress Disorder, Washington, DC, American Psychiatric Press, Inc.: 101-125.

GREEN, BL \& J.D. LINDY (1994). Posttraumatic Stress Disorder in Victims of Disasters. Psychiatric Clinics of North America, 17(2), 301-309.

HOULIAN, D., B.J. RIES, M.A. POLUSNY \& C.N. HANSON (2008). Predictors of Behavior and Level of Life Satisfaction of Children and Adolescents After a Major Tornado. Journal of Psychological Trauma, 7(1), 21-36.

HOVINGTON, C., D. MALTAIS, ET G. LALANDE (2002). Les conséquences des catastrophes sur la santé biopsychosociale des aînés: résultats de la recension des écrits, en D. Maltais (dir.) Catastrophes et état de santé des individus, des intervenants et des communautés. Saguenay, GRIR, UQAC, 189-305.

HUA, C., A. MCFARLANE \& S. KLIMIDIS (1988). Prevalence of psychiatric disorder following the 1988 Yu Pan (China) earthquake. The first 5-month period. Social

HUTCHINS, G.L. \& F.H. NORRIS (1989). Life Change in he Disaster Recovery Period, Environment and Behavior 21(1), 33-56

HUTTON, D., (2004). Psychosocial effects of a natural disaster: a post-flood assessment in the red river valley. Environments, 32(2), 27-43.

INTERNATIONAL STRATEGY FOR DISASTER REDUC TION (2004). Living with risk. A global review of disaster reduction initiatives, Geneva: United Nations publication
JONES, R.T, R. FRARY, P. CUNNINGHAM, J.D. WEDDLE \& L. KAISER (2001). The psychological effects of Hurricane Andrew on ethnic minority and Caucasian children and adolescents: a case study. Cultural diversity E ethnic minority psychology, 7(1) 103-108.

JOSEPH, L. (2006). The effects of mass trauma on children of different developmental stages: Examining PTSD in children affected by Hurricane Ivan and Hurricane Katrina. Thèse l'Université Palo Alto, Californie.

KAR, N. et al. (2007). Post-traumatic stress disorder in children and adolescents one year after a super-cyclone

KATO, H., N. ASUKAI, Y. MIYAKE, K. MINAKAWA, \& A. NISHIYAMA. (1996). Post-traumatic symptoms among younger and elderly evacuees in the early stages ollowing the 1995 Hanshin-Awaji earthquake in Japan. Acta Psychiatrica Scandinavica, 93, 477-481.

KNIGHT, B.G., M. GATZ, K. HELLER, \& V.L. BENGTSON. (2000). Age and emotional response to the Northridge earthquake: a longitudinal analysis. Psychology and Aging, $15,627-634$

KNOWLES R. \& B. GARRISON (2006). Planning for the elderly in natural disasters. Disaster Recovery Journal. 19(4).

KOHN, R. et al. (2005). Prevalence, risk factors and aging vulnerability for psychopathology following a natural disaster in a developing country. International Journal of Geriatric Psychiatry, 20(9), 835-841.

KOVATS, R.S. \& K.L EBI (2006). Heatwaves and public health in Europe. European Journal of Public Health, 16(6), 592-599.

KOZLOVSKAI. GV., A.V. BASHINA, I.P. GORIIUNOVA, E.V KIREEVA et al (1991). The Effect of the Earthquake in Armenia on the Mental Health of the Juvenile Population of the Affected Area Soviet Neurology and Psychiatry. 24(3), 11-23.

KRAUSE, N. (1987). Exploring the Impact of a Natural th and Psychological Well-being of Older Adult. Journal of Human Stress, 13, 61-69.

LABRA, O. (2013). Conséquences psychosociales chez les adultes exposés au séisme de 2010 de la ville de Talca au Chili. Collection Santé, assistance, interventions sociales et problèmes sociaux GRIR, UQAC

LACHANCE, KR, A.B. SANTOS \& B.J. BURNS (1994) Brief program Report. The Response of an Assertive

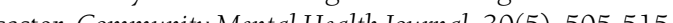

saster. Community Mental Healh Journal, 30(5), 505-515.

LACK, C.W. (2006). A comparison of posttraumatic distress related to seasonal natural disasters in exposed and non-
exposed children. Tesis de doctorado depositada en la Universidad del Estado de Oklahoma.

LALANDE, G., D. MALTAIS, ET S. ROBICHAUD (2000). Les sinistrés des inondations de 1996 au Saguenay: problèmes vécus et séquelles psychologiques. Santé mentale
au Québec, $\mathrm{XV}(1), 95-115$.

LANGLEY, A.K. \& T.J. RUSSEL (2005). Coping Efforts and Efficacy, Acculturation, and Post-Traumatic Symptomatology in Adolescents Following Wildfire, Fire Technology, 41, pp. 125-143.

LAZARATOU, H., T. PAPARRIGOPOULOS, G. GALANOS, C. PSARROS, et al. (2008). The psychological impact of a catastrophic earthquake: a retrospective study 50 years after the event. Journal Nervous Mental Disease.
196, 340-344.

LEE, I., Y.S. HA, Y.A. KIM, \& Y.H. KWON (2004). PTSD symptoms in elementary school children after Typhoon Rusa. Taehan Kanho Hakhoe chi, 34(4), 636-645.

LEWIN, T.J., V.J. CARR \& R.A. WEBSTER (1998). Recovery from Post-earthquake Psychological Morbidity :
Who Suffers and Who Recovers? Australian New Zealand Who Suffers and Who Recovers?
Journal of Psychiatry, 32, 15-20.

LOGUE, J.N., M.E. MELICK \& E.L. STRUENING. (1981). A Study of Health and Mental Health Status following a Major Natural Disaster. Research in Community and Mental Health, 2, 217-274.

LYSTAD, M. (1984). Children's Responses to Disaster: Family Implications. International Journal of Family Psychiatry, 5(1), 41-48.

MACDONNELL, S., R.P. TROIANO, N. BARKER, E. NOJI, G.W. HLADY \& R. HOPKINS (1995). Long-term Effects of Hurricane Andrew: Revisiting Mental Health Indicators, Disasters: The Journal of Disaster Studies and Management, 19(3), 235-246.

MALTAIS, D. (2003). Catastrophes en milieu rural, Saguenay, Éditions JCL, Collection Au coeur des catastrophes.

MALTAIS, D. (2007). Les personnes âgées de la Montérégie et la tempête de verglas de janvier 1997. Vie et Vieillissement, 6(2) 31-38.

MALTAIS, D. ET CÔTÉ (2007). Soutien social et personnes âgées en cas de désastre: points saillants des recherches existantes. Vie et vieillissement, 6(2), 39-42.

MALTAIS, D., N. CÔTÉ ET S. GAUTHIER (2007). Les conséquences de lexposition à une catastrophe sur la sante biopsychosociale des personnes âgées: Que savons-nous jusqu'à maintenant sur cette question? Vie et vieillissement, 6(2), 3-8.

MALTAIS, D., \& S. GAUTHIER (2010). Long Term Impacts on personal and spiritual values for French Canadian Elderly Victims of a flood in Quebec: a question of resilience. En A. Kalayjian and Eugene, D. (eds). Mass Trauma And Emotional Healing Around the World: Rituals and Practices for Resilience and Meaning-making, volume

MALTAIS D. ET S. ROBICHAUD (2009). Conséquences de la tempête de verglas en Montérégie: santé psychosociale et performance au travail des intervenants. Revue Francophone du Stress et du Trauma, 9(3), 167-176.
MALTAIS, D. (2008). La formation en situation de crise en cas de catastrophe macrosociale: une nécessité en traval social dans un monde en changement? En le cadre du Deuxième Congres International des Formateurs en Travail Social et des Professionnels Francophones de Intervention Sociale, Namur, Belgique, 3 au 7 juillet. 10 pages

MALTAIS, D. (2006). Travail social en période de catastrophe et de post-catastrophe. Revue Intervention. 125, 127-138.

MALTAIS D. RHEAULT, M-A. (2005). Lintervention sociale en cas de catastrophes. Québec, Presses de l'Universite du Québec.

MALTAIS, D., L. LACHANCE, A. BRASSARD \& M. DUBOIS (2005). Soutien social perçu, stratégies d'adaptation et état de santé psychologique post-désastre de victimes d'un désastre. Sciences Sociales et Santé, Paris, France, 23 (2), 5-38.

MALTAIS, D. L. LACHANCE, \& A. BRASSARD (2003) Satisfaction face à l'aide reçue et état de santé biopsychosociale post-désastre. Revue Canadienne de Service Social, 20(1), 39-61.

MALTAIS, D., L. LACHANCE \& BRASSARD, A. (2002) Les consequences dun sinistre sur la sante des personnes âgées de 50 ans et plus: étude comparative entre sinistrés et non-sinistrés. Revue Francophone du Stress el Du Trauma, 2(3), 147-156.

MALTAIS, D., L. LACHANCE, A. SIMARD, A. BRASSARD \& L. PICARD (2002). Difficultés et effets à long terme d'une catastrophe en milieu rural: résultats d'une étude combinant les approches qualitative et quantitative. Revue Québécoise de Psychologie, 23(2), 197-217.

MALTAIS, D., S. ROBICHAUD \& A. SIMARD (2001). Consequences des inondations de juillet 1996 sur la conception du chez-soi et la santé biopsychosociale des préretraités et retraités. Revue Canadienne du Vieillissement, 20(3), 407-426.

MALTAIS, D. ET N. SIMARD (2008). Les effets à long terme de l'exposition à une catastrophe sur la santé biopsychosociale des individus, en D. Maltais (dir.) Intervention Sociale en Cas de Catastrophe, Canada PUQ, 169-183.

MARTIN, C., K. BEYER, L. COLBEAU-JUSTIN, M. DEVAUX et al. (2010). Le séisme du Chili MW=8.8 du 27 Février 2010. Rapport de la Mission AFPS/SGEB, Associatio Française du Génie Parasismique, $171 \mathrm{p}$.

MCFARLANE, AC. (1995). Helping the Victimes of Disaster heory to Practice, New York, Plenum Press, 287-314.

MILLER, M.L. (2012). Controlling Disasters: Recognizin Latent Goals in Katrina's Aftermath. Journal of Disaster Studies, Policy and Management, 36, 122-139.

MILLER, J.A., J.G. TURNER \& E. KIMBALL (1981) Big Thompson Flood Victims: One Year Later. Family Relations, 30, 111-116. 
NEWMAN, C.S. (1976). Children of Disaster: Clinical Observations at Buffalo Creek. American Journal of Psychiatry, 133, 306-312.

NIGG, J.M., K.J. TIERNEY (1993) Disasters and Social Changes. Ed. American Sociological Association. Miami Florida.

NG, C., H. MA et al. (2009). China-Australia training on psychosocial crisis intervention: response to the earthquake shiatry, 17(1), 51-5

NORRIS, F. H. et al. (2002). Placing Age Differences in Cultural Context: A Comparison of the Effects of Age on PTSD After Disasters in the United States, Mexico, an
Poland. Journal of Clinical Geropsychology, 8, (3) 153-173.

NORRIS, F.H, F. PHIFER, \& K. KANIASTY (1990). NORRIS, F.H, F. PHIFER, \& K. KANIASTY (1990)
Individual and Community Reactions to the Kentucky Individual and Community Reactions to the Kentucky Floods: Finding from a Longitudinal Study of Older
Adults, en RJ Urbano BG, McCaughey y CS, Fullerton Adults, en R U Urbano BG, McCaughey y CS, Fullerto
(editores), Individual and Community Responses to Traum and Disaster: The Structure of Human Vhaos (370-400) and Disaster: The Structure of

NORRIS, F.H \& S. MURRELL. (1988). Prior experience as moderator of disaster impact on anxiety symptoms in older adults. American Journal of Community Psychology, 16, 665-683.

OLIVER-SMITH, A. (1990). Post-disaster housing reconstruction and social inequality: a challenge to policy and practice. Disasters, 14, 7-19.

OLLENDICK, D.G. \& S.M. HOFFMAN (1982). Assessmen of Psychological Reactions in Disaster Victims. Journa of Community Psychology, 10, 157-167.

OLSON, L, (1993). After the Flood: The Dripping Faucet Syndrome. Journal of the Medical Society, 83(9), 324-328.

OSAKI, Y. \& M. MINOWA (2000). Factors Associated with Earthquake Deaths in the Great Hanshin-Awaj Eartquake, 1995. American Journal of Epidemiology, 153(2), 153-156.

RAPHAEL B. (1975). Crisis and Loss: Counseling following a Disaster. Mental Health in Australia, 118-122.

ROBICHAUD, S., D. MALTAIS, G. LALANDE, A. SIMARD et al. (2001). Les inondations de juillet 1996: une série devenemements stressants, en D. Maltais (dir.) Catastrophes et elar de sanse des, en D. Mis, des inlervenanls et des communautés. Saguenay, GRIR, UQAC, pp. 101-120.

ROBICHAUD, S., D. MALTAIS, G. LALANDE, A. SIMARD et al. (2001). Les inondations de juillet 1996: une série d'événements stressants. Service Social, 48, 16-33.

RODRÍGUEZ, H., J. BARNSHAW (2006). The socia construction of disasters: From heat waves to worstcase scenarios. Cotemporary Sociology. 35(3), 218-222.

ROSENKOETTER, M.M., E.K. COVANEK, B. COBB, S. BUNTING et al. (2007). Perceptions of older adults regarding evacuation in the event of a natural disaster. Public Health Nursing. 24, 160-168).
RUSSONIELLO, C.V. et al. (2002). Childhood posttraumatic stress disorder and efforts to cope after Hurricane Floyd Behavioral medicine, 28(2), 61-71.

PENG, K., H. SHUCHENG et al. (2009). Prevalence and risk factors for posttraumatic stress disorder: a cross-sectional study among survivors of the Wenchuan 2008 earthquake in China. Depression \& Anxiety, 26, (12), 1134-1140.

PHIFER, J.F. (1990). Psychological Distress and Somatic Symptoms after Natural Disaster: Differential Vulnerability among Older Adults. Psychology and Aging, 5(3), 412-420.

PHIFER, J.F. (1988). The Impact of Natural Disaster on the Health of Older Adults: A Mutiwave Study. Journal of Health and Social Behaviour, 29, 65-78.

PHIFER, J.F. \& F.H. NORRIS (1989). Psychological Symploms in Older Adults following Natural Disaster: Nature, Timing, Duration and Course. Journal of Gerontology,

PULLINS, L.G. et al. (2005). School-Based Post-Flood Screening and Evaluation: Findings and Challenges in
One Community. Stress, Trauma, and Crisis, 8(4), 229-249.

PYNOOS, J.T. \& K. NADER (1993). Issues in the Treatment of Posttraumatic Stress in Children and Adolescents, in J.P. Whison et B. Raphael (Eds.) International Handbook of Traumatic Stress Syndrones, New York and London,

SCHEERINGA, M.S. \& C.H. ZEANAH (2008). Reconsideration of Harm's Way. Onsets and Comorbidity Patterns of Disorders in Preschool Children and Their Caregivers Following Hurricane Katrina. Journal of Clinical Child and Adolescent Psychology, 37(3), 508-518.

SCHININÀ, G, M.A. HOSN, A. ATAYA, K. DIEUVEUT $e t$ al. (2010). Psychosocial response to the Haiti earthquake: the experiences of International Organization. Intervention, 8(2). 158- 164.

SHELBY, JS \& MG TREDINNICK (1995). Crisis Intervention with Survivors of Natural Disasters and Post-disaster Mental Health Services, Journal of Community Psychology, $10,22-28$.

SMITH, B.W. (1996). Coping as a predictor of outcomes following the 1993 Midwest flood, Journal of Social Behavior and Personality, 11(2), 225-239.

SOLOMON S.D., E.M. SMITH, L.N. ROBINS, R.L. FISCHBACK (1987). Social Involvement as a Mediator Disasters-induced Stress. Journal of Applied Social Psycho$\log , 17,1092-1112$

SPIEGEL A. (2006). Katrina's Impact on Elderly Still Resonates. National Public Radio: http./Www nprorg/ templates/story.php?storyld $=5239019$

STEPHENS, K.U., D. GREW, K. CHIN, P. KADETZ et al. (2007). Excess mortality in the aftermath of Hurrican Katrina: a preliminary report. Medical Public Health Preparation, 1(1), 15-20.
STOCK, P. (2007). Katrina and Anarchy: A Content Analysis of a New Disaster Myth. Sociological Spectrum, 27(6), 705-726

STREETER ET S.A. MURTY, Eds. (1996). Research on Social Work and Disasters, New York, The Haworth Press.

SUNDET, P. \& J. MERMELSTEIN (1996). Predictors of Rural Community Survival after Natural Disaster: Implications for Social Work Practice. Research on Socia Work and Disaster, 22(1/2), 57-70.

TANIDA, N. (1996). What Happened to Elderly People in the Great Hanshin Earthquake, British Medical Journal, 113, 1133-1135

THOMPSON, M.P., F.N. NORRIS \& B. HANACEK (1993), Age Differences in the Psychological Consequences of Hurricane Hugo. Psychology and Aging, 8(4), 606-616

TICEHURST, S., R.A. WEBSTER, V.J. CARR \& T.J. LEWIN (1996). The Psychosocial Impact of an Earthquake on 11(11), 943-951

TIERNEY K. (2006). Foreshadowing Katrina: Recent sociological contributions to vulnerability science. Contemporary Sociology. 35(3), 207-212.

TYLER, K.A. \& D.R. HOYT (2000). The Effects of an Acute Stressor on Depressive Symptoms among Older Adults. Research on Aging, 22(2), 143-164.

UDOMRATN P. (2008). Mental health and the psychosocial consequences of natural disasters in Asia. International Review of Psychiatry, 20(5), 441-444.

VAN DER KOLK, E.M., W.K. SILVERMAN, A.M. LAGRECA \& M.J. PRINSTEIN (1996). Prediction of Postraumatic Stress Symptoms in Children after Hurricane Andrew. Journal of Abnormal Psychology, 105(2), 237-248.

WANG, P.S \& AL. (2007). Mental health service use among hurricane Katrina survivors in the eight months after the disaster. Psychiatric services, 58(11), 1403-1411.

WANG, X., L. GAO \& AL. (2000). Post-earthquake quality of life and psychological well-being: Longitudinal evaluation in a rural conmunity sample in northern China. Psychiatry \& Clinical Neurosciences, 54(4), 427-433.

WEINTRAUB, D \& P.E. RUSKIN (1999). Posttraumatic Stress Disorder in the Elderly: A Review. Harvard Review of Psychiatry, 7(3), 125-183.

WILBANKS T., ROMERO LANKAO P., BAO M., BERKHOUT F., CAIRNCROSS S., CERON J.-P., KAPSHE M., MUIRWOOD R., \& ZAPATA-MARTI R. (2007). Industry, settlement and society, en M. Parry, O. Canziani, J. Palutikof, P. van der Linden et C. Hnason (eds.), Climate Change 2007: Impacts, Adaptation and Vulnerability, contribution du Groupe de travail II au Quatrième rapport devaluation du Groupe dexperts intergouvernementaux sur l'evolution du clima, Cambridge, Royaume-Uni Cambridge University Press, 357-390.
ZAKOUR, M.J. \& E.B. HARREL (2003). Access to Disaste Services: Social Work Interventions for Vulnerable Populations. Journal of Social Service Research, 30(2), 27-54

ZAKOUR, M.J. (1996). Disaster Research in Social Work, en C.L. Streeter et S.A. Murty (Eds), Research on Socia Work and Disasters, New York, The Haworth Press, 7-27. 